\title{
Chicoric acid is a potent anti-atherosclerotic ingredient by anti- oxidant action and anti-inflammation capacity
}

\author{
Kun-Ling Tsai ${ }^{1, *}$, Chung-Lan Kao ${ }^{2, *}$, Ching-Hsia Hung ${ }^{1,3, *}$, Yung-Hsin Cheng ${ }^{4}$, Huei- \\ Chen Lin ${ }^{2,5}$ and Pei-Ming Chu ${ }^{6}$ \\ ${ }^{1}$ Department of Physical Therapy, College of Medicine, National Cheng Kung University, Tainan, Taiwan \\ 2 Department of Physical Medicine and Rehabilitation, Taipei Veterans General Hospital, Taipei, Taiwan \\ ${ }^{3}$ Institute of Allied Health Sciences, College of Medicine, National Cheng Kung University, Tainan, Taiwan \\ ${ }^{4}$ Department of Education and Research, Taipei City Hospital, Taipei, Taiwan \\ ${ }^{5}$ Department of Physical Therapy, Shu-Zen Junior College Of Medicine And Management, Kaohsiung, Taiwan \\ ${ }^{6}$ Department of Anatomy, School of Medicine, China Medical University, Taichung, Taiwan \\ ${ }^{*}$ These authors have contributed equally to this work
}

Correspondence to: Pei-Ming Chu, email: pmchu@mail.cmu.edu.tw

Keywords: atherosclerosis, free radical, antioxidant, chicoric acid, inflammation, Gerotarget

Received: January 25, $2016 \quad$ Accepted: March 04, $2017 \quad$ Published: March 31, 2017

Copyright: Tsai et al. This is an open-access article distributed under the terms of the Creative Commons Attribution License (CC-BY), which permits unrestricted use, distribution, and reproduction in any medium, provided the original author and source are credited.

\section{ABSTRACT}

Atherosclerotic cardiovascular disease is linked to both oxidative stress and endothelial cell dysfunction. Chicoric acid has antioxidant and anti-inflammatory properties. In the present investigation, we demonstrated that chicoric acid inhibits oxidized low-density lipoprotein (oxLDL)-facilitated dysfunction in human umbilical vein endothelial cells (HUVECs). Oxidative injuries were tested by investigating the formation of intracellular reactive oxygen species (ROS) and by examining the activity of antioxidant enzymes and the function of endothelial nitric oxide synthase (eNOS). We also confirmed that chicoric acid mitigates apoptotic features caused by oxLDL, such as the subsequent break down of mitochondrial transmembrane potential and the activation of Bax, which promote DNA strand breaks and activate caspase-3. Moreover, our data revealed that chicoric acid attenuated the oxLDL activation of NF-KB, the attachment of THP-1 cells and the overexpression of adhesion molecules in human endothelial cells. The results of this study suggest a potential molecular mechanism through which chicoric acid inhibits oxLDL-induced human endothelial dysfunction.

\section{INTRODUCTION}

Atherosclerotic damage is linked to oxidative injuries [1]. The initial stages of atherosclerosis are facilitated by the augmentation of oxidized low-density lipoprotein (oxLDL) and the aggregation of vascular cells. The subsequent overexpression of adhesion molecules and enhanced adherence of monocytes to endothelial cells were shown as important events in the progression of atherosclerosis $[2,3]$. The oxLDL-induced endothelial toxicity and morphological changes observed in cultured endothelial cells are alike to those detected in animal in endothelial cells overlying atherosclerotic areas [4].
oxLDL-induced reactive oxygen species (ROS) formation plays a vital role in the mediation of endothelial dysfunction and thus regulate endothelial apoptosis as well as inflammation [5, 6]. High doses of ROS can cause cell dysfunction and death via the oxidation-induced modification of proteins, nucleic acids, carbohydrates and lipids. Those modifications consequently regulate endothelial inflammatory responses and apoptotic events [7]. Moreover, these pro-apoptotic responses are related to disturbances in mitochondrial membrane permeability and the subsequent release of cytochrome c, and the upregulation of executioner caspases [8]. The presence of activated NF- $\mathrm{NB}$ in atherosclerotic lesion suggests a 
potential role of NF- $\mathrm{KB}$ in atherosclerotic pathology; a local inflammatory even is associated in atherosclerotic pathology, and NF- $\mathrm{kB}$ is shown to be involved in this process [9-11]. NF- $\mathrm{KB}$ also mediates the function of various genes involved in the balance between cell survival and apoptosis [12]. E- and P-selectin, vascular cell adhesion molecule-1 (VCAM-1) and intercellular cell adhesion molecule-1 (ICAM-1) are adhesion molecules in human endothelial cells. Moreover, NF- $\mathrm{kB}$ plays a positive role in the activation of those adhesion molecules. In addition, adhesion molecules are thought to be early indicators of atherosclerosis [13]. For this reason, therapeutic strategies involving inhibitors of oxLDL-mediated endothelial dysfunction may reduce the progression of atherosclerotic pathology, inhibit their morbidity and promote the survival of patients with cardiovascular diseases.

Chicoric acid is one compound which could be obtained from isolated and purified plant and vegetables. In addition, chicoric acid has been reported as one daily nutraceutical to enhance antioxidant activity [14]. Tousch et al suggested chicoric acid is one novel ingredient able to increase insulin release as well as glucose uptake. Chicoric acid was also shown to facilitate 3T3-L1 preadipocytes dysfunction by modulation of ROS-mediated PI-3K/AKT and MAPK mechanism [15]. Therefore, chicoric acid is described as having potential anti-diabetic and antiobesity capacities. Landmann et al reported chicoric acid mitigates acute alcohol-induced hepatic steatosis in mice through oxidative stress inhibition and anti-inflammation properties [16].Liu et al reported that chicoric acid intervention protects against systemic inflammationcaused memory impairment and amyloidogenesis through inactivation of NF- $\mathrm{KB}$, indicating that chicoric acid has positive effects in management of chronic systemic diseases [17]. In contrast to the previously mentioned studies of the in vivo and in vitro antioxidant and antiinflammatory effects of chicoric acid, no investigations were performed on chicoric acid effects on oxLDL-caused oxidative injuries in human endothelial cells.

Therefore, in this study, we explored whether chicoric acid inhibits oxLDL-mediated endothelial injuries and attempted to prove the cytoprotective effects of Chicoric acid, which prevent the oxLDL-mediated inhibition of endothelial NO synthase (eNOS), the overexpression of oxLDL-induced adhesion molecules and the monocyte adhesion. Additionally, we also investigated several apoptotic events, including mitochondrial destabilization and the up-regulation of caspases.

\section{RESULTS}

\section{Chicoric acid reduces oxLDL-facilitated ROS generation in HUVECs}

ROS act a key role in regulation of pro-apoptotic signal transduction pathways and cell death [18]. Therefore, we initially studied the effects of chicoric acid on the formation of ROS, a potential candidate related to oxLDL-promoted endothelial dysfunction, by DCF-AM. The chemical structure of chicoric acid was revealed in Figure 1. Pretreatment of endothelial cells with chicoric acid $(12.5-100 \mu \mathrm{M})$ for $2 \mathrm{~h}$ before stimulate with oxLDL inhibited the formation of ROS (all $\mathrm{P}<0.05$ ) (Figure 2A, 2B). Moreover, ROS are known to attenuate antioxidant enzymes, promoting an imbalance that favors oxidative stress [19]. Therefore, we tested the activity of both superoxide dismutase (SOD) and catalase in oxLDLexposed endothelial cells [5]. Our results revealed that oxLDL induced ROS formation but not inhibited SOD and catalase activity in $2 \mathrm{~h}$ treatment. However, oxLDL reduced SOD and catalase activity in $24 \mathrm{~h}$ treatment (data not show). This result suggested that oxLDL-induced ROS formation may the up-stream signaling in regulation of cell death and antioxidant enzymes dysfunction. However, In Figure 2C and 2D, we show that endothelial SOD and catalase activity was significantly reduced after exposure to oxLDL. These results were effectively reversed by chicoric acid treatment.

\section{Chicoric acid reverses oxLDL-inhibited eNOS phosphorylation}

A previous report suggested that the secretion of NO from HUVECs is inhibited by oxLDL, facilitating cell

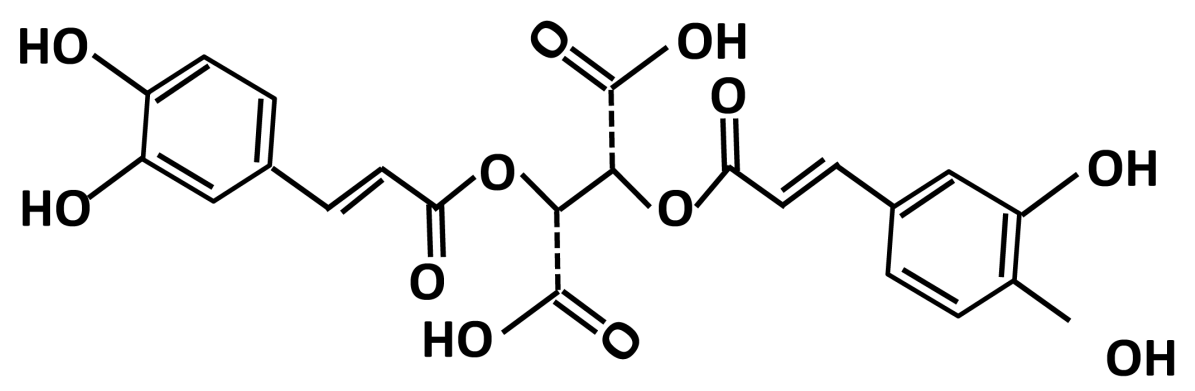

Figure 1: The chemical structure of chicoric acid. 
death in human endothelial cells [20]. However, whether chicoric acid protects endothelial cells from oxLDLinhibited eNOS phosphorylation remained unknown. Therefore, we tested the effects of chicoric acid on eNOS phosphorylation. As shown in Figure 3A and 3B, oxLDL largely inhibited eNOS protein phosphorylation in endothelial cells after $24 \mathrm{~h}$ of stimulation; this result was significantly reversed by chicoric acid intervention.

\section{Effects of chicoric acid on oxLDL-promoted apoptosis}

Annexin $\mathrm{V}$ was used to determine both the proapoptotic effects of oxLDL and the anti-apoptotic ability of chicoric acid on endothelial cells. Flow cytometric analysis demonstrated that apoptotic cells (20.3\%) and necrotic cells $(8.4 \%)$ were found in endothelial cells stimulated with oxLDL. The apoptotic cells in HUVECs pretreated with $100 \mu \mathrm{M}$ chicoric acid (8.8\%) was similar to that observed in control sample (Figure 4A). Moreover, the opening of the mitochondrial permeability transition pore (PTP) has been suggested to play a vital role in the pathway that promotes apoptosis. The mitochondrial membrane potential $(\Psi \mathrm{m})$ collapses by both the disability of the electrochemical gradient caused via pore opening and the rupture of the outer mitochondrial membrane [21]. We subsequently investigated mitochondrial permeability to determine whether chicoric acid inhibits mitochondrial stability after stimulate with oxLDL. As shown in Figure $4 \mathrm{~B}$, oxLDL depolarized the mitochondrial membrane in endothelial cells, as demonstrated by the up-regulation in FL1 (middle panel), Intervention with chicoric acid resulted in stability of the mitochondrial transmembrane potential, as indicated by both the reduction of FL1 and the reversion $n$ of FL2 (right panel).
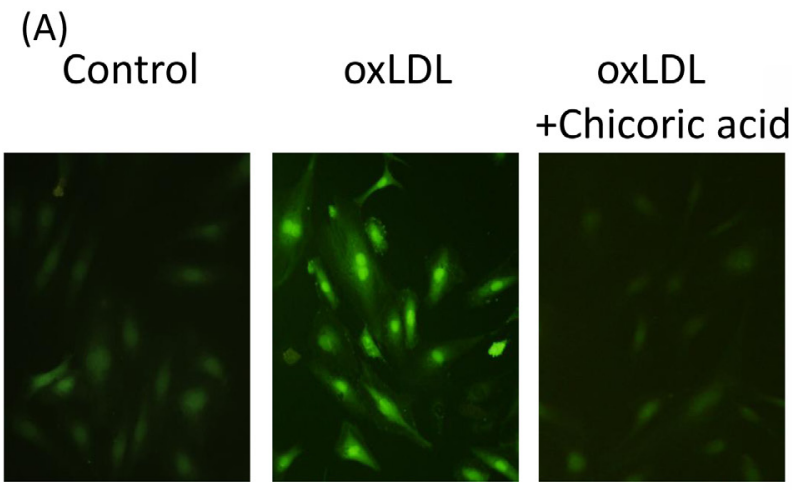

(C)

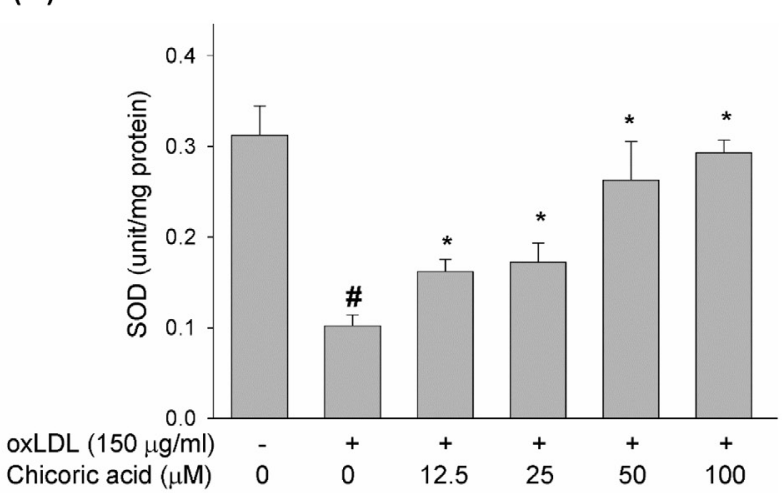

(B)

(D)
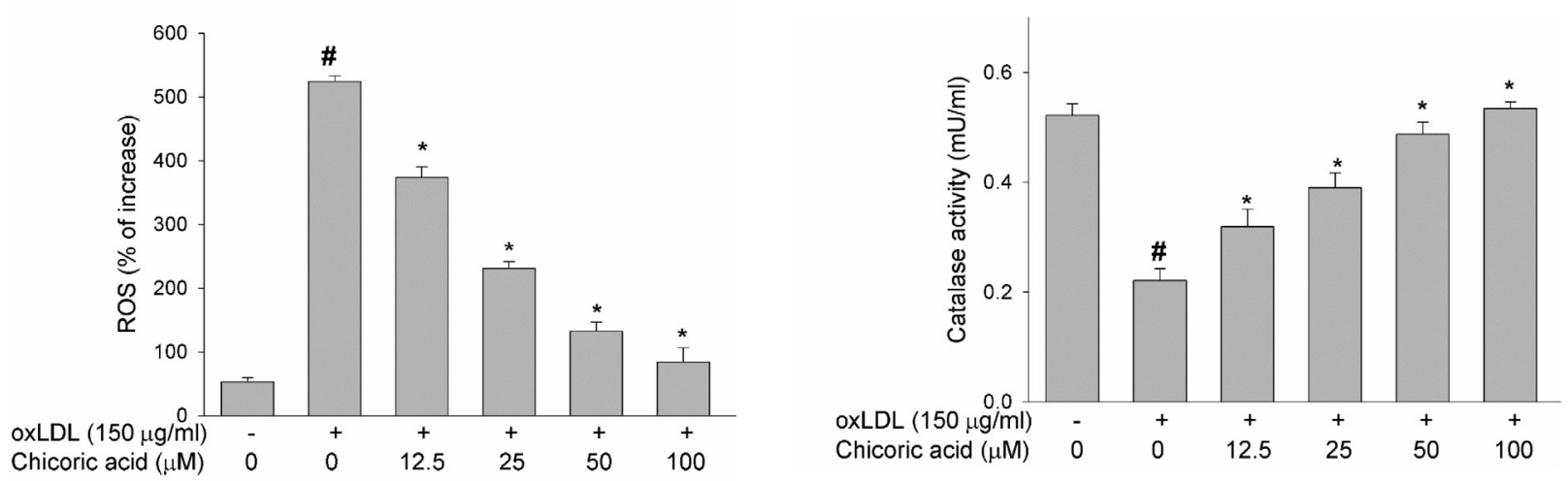

Figure 2: The inhibitory effects of chicoric acid on oxLDL-facilitated ROS generation in endothelial cells. After pretreatment for $2 \mathrm{~h}$ with the indicated concentrations of chicoric acid (12.5-100 $\mu \mathrm{M})$, HUVECs were incubated with the $\mathrm{H}_{2} \mathrm{O}_{2}$-sensitive fluorescent probe DCF-AM $(10 \mu \mathrm{M})$ for $1 \mathrm{~h}$, followed by treatment with $150 \mu \mathrm{g} / \mathrm{ml}$ oxLDL. A. Fluorescence images revealed the ROS level in control cells (left) and HUVECs stimulated with oxLDL (middle) in the presence of $100 \mu \mathrm{M}$ chicoric acid (right). B. Fluorescence intensity of HUVECs was measured with a fluorescence microplate reader. Fluorescence distribution of DCF-AM oxidation was expressed as a percentage of increased intensity. The activity of $\mathbf{C}$. SOD and D. catalase in HUVECs stimulated with oxLDL in the absence or presence of indicated concentrations of chicoric acid were determined. Data are expressed as the mean \pm SD. of three independent analyses. $\# P<0.05$ vs. untreated control; ${ }^{*} P<0.05$ compared with oxLDL treatment. 
Anti-apoptotic genes in the Bcl-2 family mediate mitochondrial outermembrane permeability and able to act as either an anti-apoptotic c or a pro-apoptotic regulator. The activation of the pro-apoptotic protein p53 is thought to be an important process in oxLDL-induced endothelial cell death via the promotion of ROS generation and subsequent induction of a conformational change in Bax that empowers the mitochondrial translocation of proapoptotic events [22]. We used a Western blotting assay to test the protective effects of chicoric acid against the oxLDL-mediated activation of Bax and the inhibition of Bcl-2. In Figure 5A, 5B, we confirmed that chicoric acid both significantly mitigated the activation of Bax and reversed the expression of Bcl-2. Caspase- 3 also acts a vital role in mitochondrial apoptosis [21]. To test whether chicoric acid inhibits oxLDL-activated caspase-3 activation, we validated the active form of caspase- 3 using a flow cytometry assay. In Figure 5C, the activity of caspase-3 enriched in endothelial cells exposed to oxLDL. However, the oxLDL-activated caspase-3 was mitigated in cells pretreated with $100 \mu \mathrm{M}$ chicoric acid. In order to determine whether that oxLDL-caused cell death is an apoptotic response, oxLDL-treated cells were analyzed biochemically via flow cytometry using a TUNEL assay. As shown in Figure 5D, the results of indicated that chicoric acid is a potent repressor of oxLDL-caused cytotoxicity in HUVECs.

\section{Chicoric acid reduces the oxLDL-induced cytotoxicity of endothelial cells}

At the end of stimulation with oxLDL, the viability of HUVECs exposed with oxLDL in the absence or

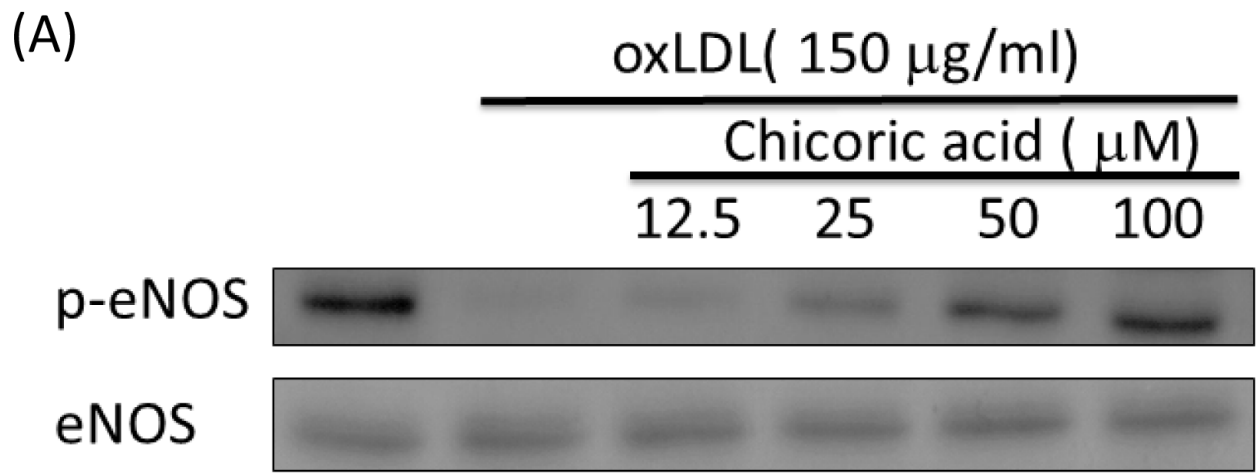

(B)

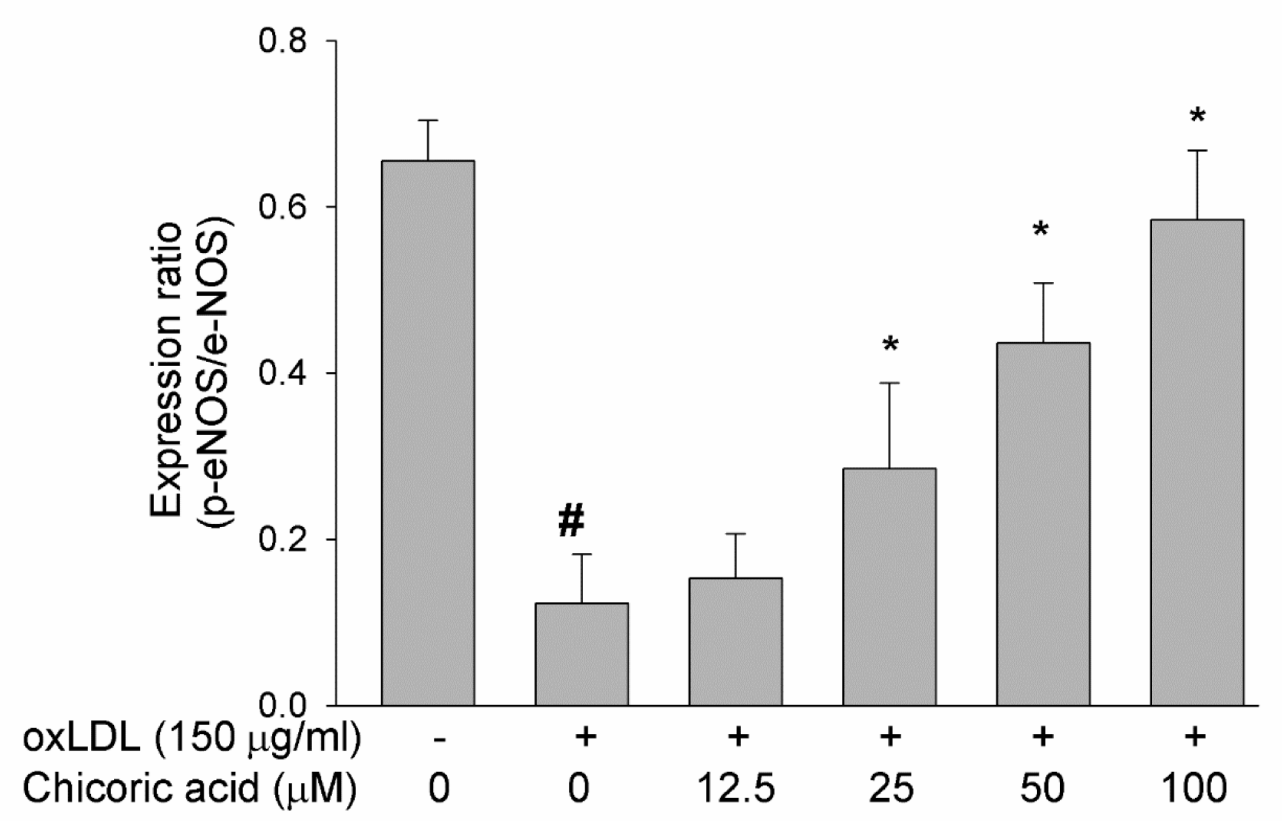

Figure 3: Effects of chicoric acid on oxLDL-repressed eNOS protein phosphorylation. HUVECs were pretreated for 2 hours with the indicated concentrations of chicoric acid (12.5-100 $\mu \mathrm{M})$, followed by oxLDL for 24 hours. A.,B. For Western blot analyses, a monoclonal anti-p-eNOS (Ser1177) and a monoclonal anti-eNOS antibody (for normalization) were used. The values represent means \pm SD error from three separate experiments. \# $P<0.05$ vs. untreated control; $* P<0.05$ compared with oxLDL treatment. 
presence of chicoric acid was studied by MTT assay, and the membrane permeability was evaluated via LDH concentration assay. In Figure 6, our data revealed that oxLDL reduced cell viability and enhanced membrane permeability in HUVECs after $24 \mathrm{~h}$ of exposure; however, pretreatment with chicoric acid attenuated the oxLDLinduced cytotoxicity in HUVECs in a dose-dependent manner. Besides, we also confirmed the SNP (NO donor) definitely reduced oxLDL-caused cell death, confirming that eNOS function may act a key role in regulation of oxLDL-induced cell death.

\section{Chicoric acid reduces oxLDL-mediated p38 MAPK and NF- $\kappa$ B activation}

The ROS produced by oxLDL were shown to upregulate $\mathrm{p} 38$ MAPK and down-regulate phosphoinositide 3-kinase (PI3K). Furthermore, both of these signaling pathways lead to the activation of NF- $\kappa \mathrm{B}$, which subsequently regulates downstream pro-inflammatory events [23]. As revealed in Figure 7A, the oxLDL promoted p38 MAPK phosphorylation was mitigated in endothelial cells pretreated with chicoric acid.

$\mathrm{NF}-\kappa \mathrm{B}$ is a family of dimers composed of members of the Rel/NF- $\kappa$ B family [24]. NF- $\kappa$ B activation needs the dissociation of the repressor $\mathrm{I}-\kappa \mathrm{B}$. Once this dissociation occurs, NF- $\kappa \mathrm{B}$ is immediately translocated to the cell nucleus, in which it is a p65/p50 heterodimer and binds to its cognate sequence of DNA [25]. Figure 7C and 7D shown that I- $\kappa$ B decreased after stimulation with oxLDL, thereby facilitating the nuclear translocation of NF- $\kappa$ B p65. However, in HUVECs pretreated with chicoric acid, this phenomenon is effectively inhibited.

\section{Chicoric acid inhibits the oxLDL-caused attachment of THP-1 cells to endothelial cells and the activation of adhesion molecules}

Kim et al. reported that oxLDL-increased the retention, recruitment, and adhesiveness of human monocytes to the endothelium, a process associated with the earliest stages of atherogenesis [26]. To test
(A)

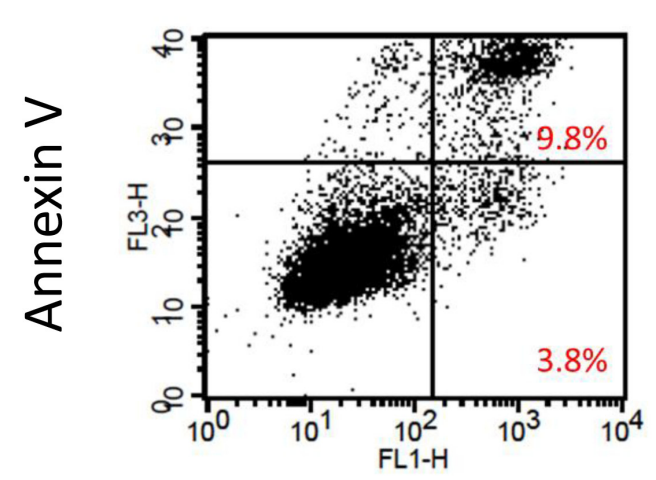

(B)

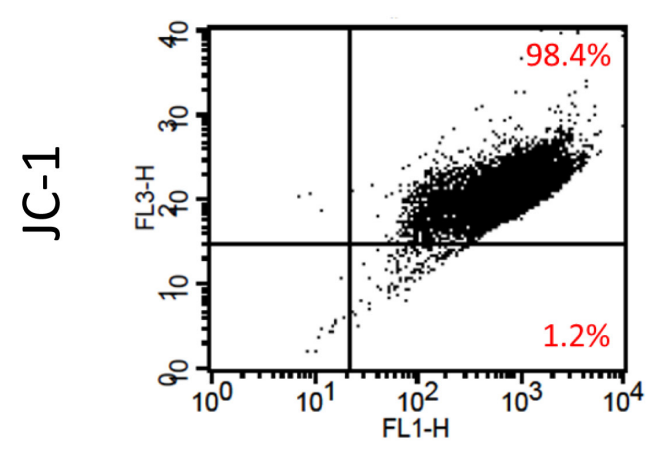

Control
Control

Figure 4: Chicoric acid blocked oxLDL-induced apoptosis in HUVECs. A. HUVECs were incubated with oxLDL (150 $\mu$ g/ $\mathrm{ml}$ ) in the absence or presence of indicated concentrations of chicoric acid for $24 \mathrm{~h}$. Apoptotic and necrotic death of oxLDL-exposed endothelial cells were tested by a FITC-labeled annexin V assay and PI staining. Flow cytometry was used for confirmation. B. $\Delta \Psi \mathrm{m}$ was inspected with the signal from monomeric and J-aggregate JC-1 fluorescence, as described in the Materials and Methods. (left) No treatment; (middle) oxLDL; (right) oxLDL + chicoric acid. JC-1 fluorescence was confirmed by flow cytometry. 
the inhibitory effects of chicoric acid against monocyte adhesion to HUVECs, confluent monolayers of endothelial cells were treated with chicoric acid for $2 \mathrm{~h}$ and then stimulated with oxLDL for a further $24 \mathrm{~h}$, followed by co-culture with THP- 1 cells for $1 \mathrm{~h}$ in $37^{\circ} \mathrm{C}$. Our results demonstrate that oxLDL increased the attachment of THP1 cells in endothelial cells (Figure 8A). This outcome was significantly mitigated by chicoric acid intervention. The inhibitory effects of chicoric acid on adhesion molecules in endothelial cells stimulated by oxLDL were confirmed next. Figure 8B-8D, we found the expression levels of ICAM-1, VCAM-1, and E-selectin were obviously higher in endothelial cells exposed to oxLDL for $24 \mathrm{~h}$ than in the control samplegroup. Flow cytometry suggested that adhesion molecule expression was repressed by chicoric acid.

\section{DISCUSSION}

It has been reported that oxidative injuries induced by oxLDL triggers the signaling pathways causing to cellular death. Furthermore, ROS formation serves as the initial pro-apoptotic signal of oxLDL, thereby activating multiple downstream pathways [1]. In our study, we have shown that chicoric acid ameliorates oxLDLfacilitated dysfunction in endothelial cells by repressing inflammatory responses and oxidative damage. Chicoric acid inhibited the production of ROS, which subsequently inhibited the function of both SOD and catalase, promoted the bioavailability of NO and maintained the mitochondrial membrane, thereby preventing pro-apoptotic responses in oxLDL-treated endothelial cells.

In normal cells, the balance between ROS formation and the activity of antioxidant enzyme is maintained [27]. There are different types of ROS produced in endothelial cells, including the hydrogen peroxide $\left(\mathrm{H}_{2} \mathrm{O}_{2}\right)$, superoxide $\left(\cdot \mathrm{O}_{2}\right)$, peroxynitrite $(\cdot \mathrm{ONOO})$, hydroxyl $(\cdot \mathrm{OH})$ radicals and NO. The intracellular ROS concentration is constant as a result of the homeostasis between ROS-generating enzymes and antioxidant enzymes; however, the function of these antioxidant enzymes is inhibited by $\mathrm{H}_{2} \mathrm{O}_{2}$, which is produced by the dismutation of the superoxide
(A)

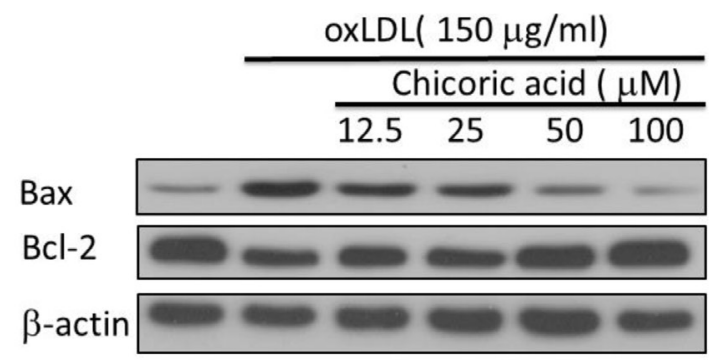

(B)

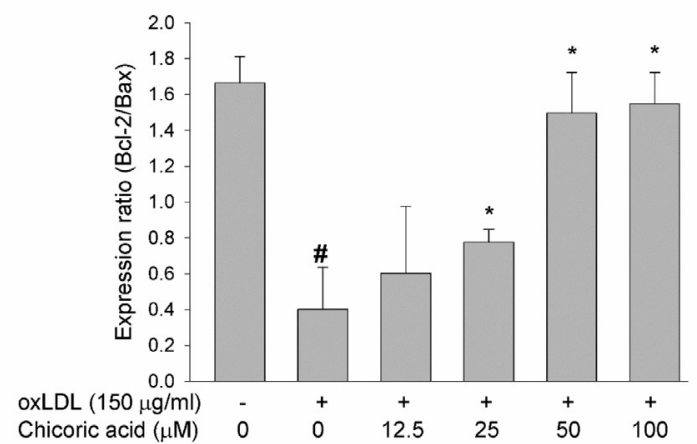

(C)

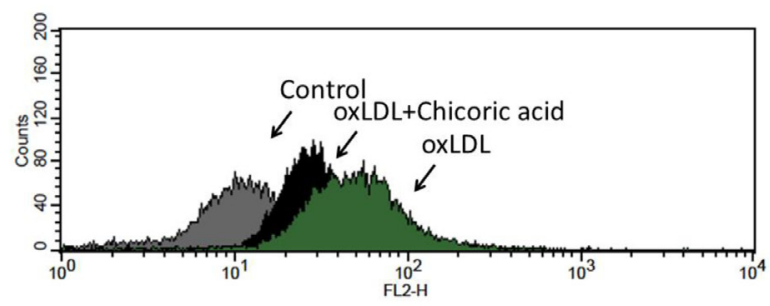

(D)

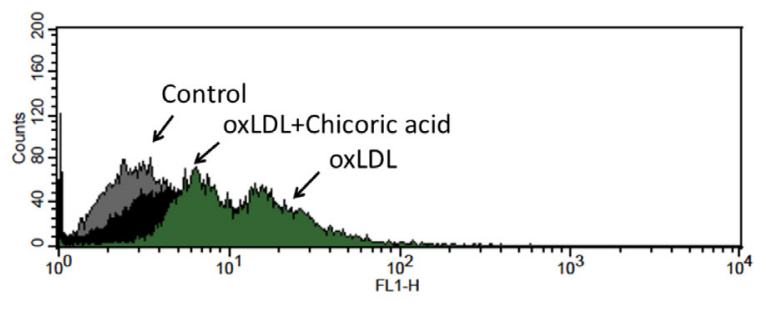

Figure 5: Effects of chicoric acid on oxLDL-triggered endothelial apoptosis. A. Immunoblotting analysis of apoptotic cells in HUVECs exposed to oxLDL and chicoric acid. HUVECs were incubated with $150 \mu \mathrm{g} / \mathrm{ml}$ oxLDL in the absence or presence of indicated concentrations (12.5-100 $\mu \mathrm{M})$ of chicoric acid for $24 \mathrm{~h}$. Representative Western blots and summary data showing that oxLDL up-regulated Bax and down-regulated Bcl-2 proteins. Intervention with chicoric acid suppressed the above mentioned oxLDL-induced responses. Results were confirmed by densitometric analysis B.; the values are presented as means $\pm \mathrm{SD}$ of three separate experiments. $\# P<0.05 v s$. untreated control; ${ }^{*} P<0.05 v s$. oxLDL treatment. C. Effect of chicoric acid on oxLDL-induced caspase-3 activation. HUVECs were incubated with oxLDL in the absence (right) and presence (middle) of $100 \mu \mathrm{M}$ chicoric acid. Activated of caspase- 3 was examined by using flow cytometry (control: grey; oxLDL: black; oxLDL with chicoric acid: green). D. Effect of chicoric acid on oxLDL-induced DNA damage. HUVECs were incubated with oxLDL in the absence (right) and presence (middle) of $100 \mu \mathrm{M}$ chicoric acid. TUNEL assay was examined by using flow cytometry (control: grey; oxLDL: black; oxLDL with chicoric acid: green). 
(A)

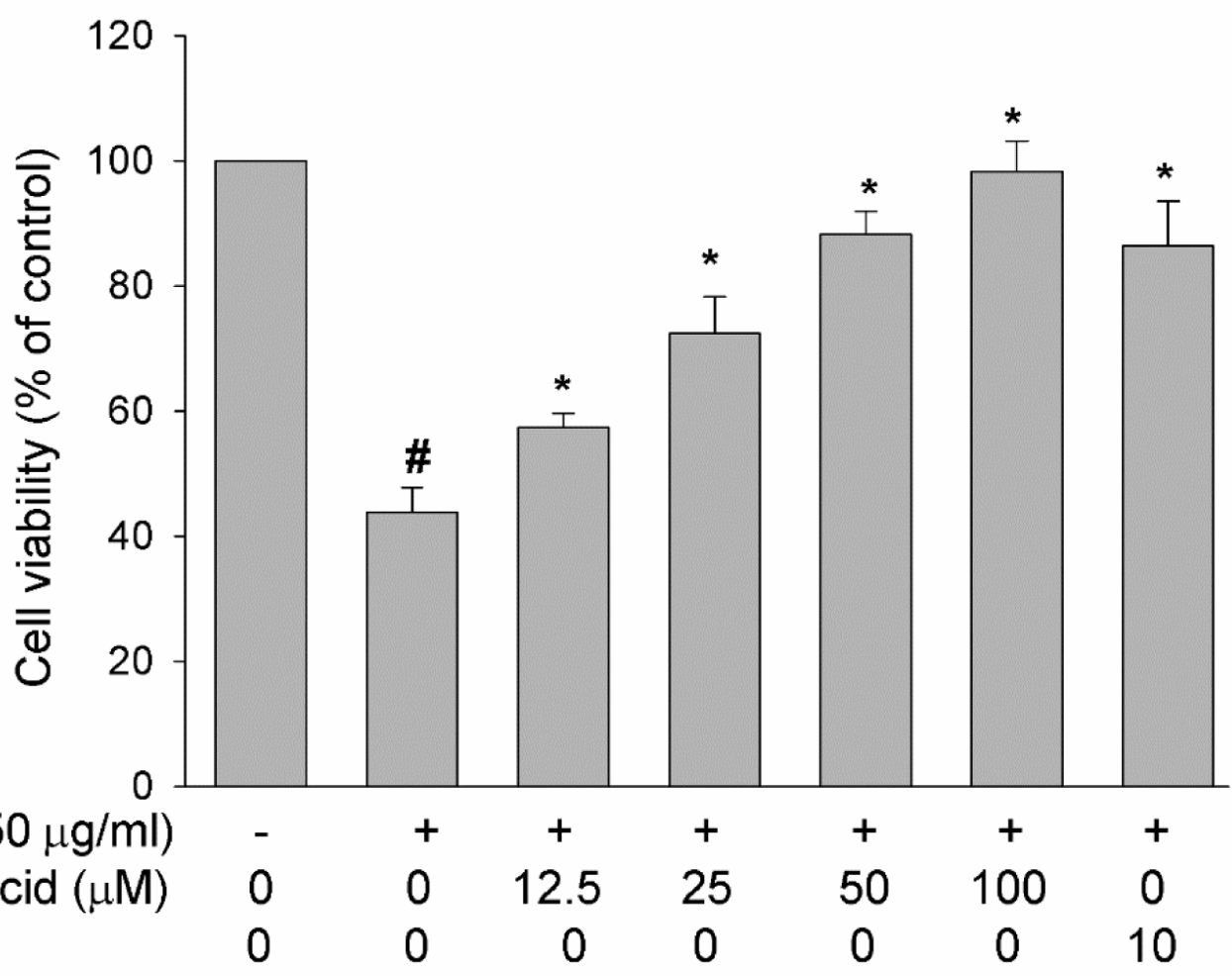

(B)

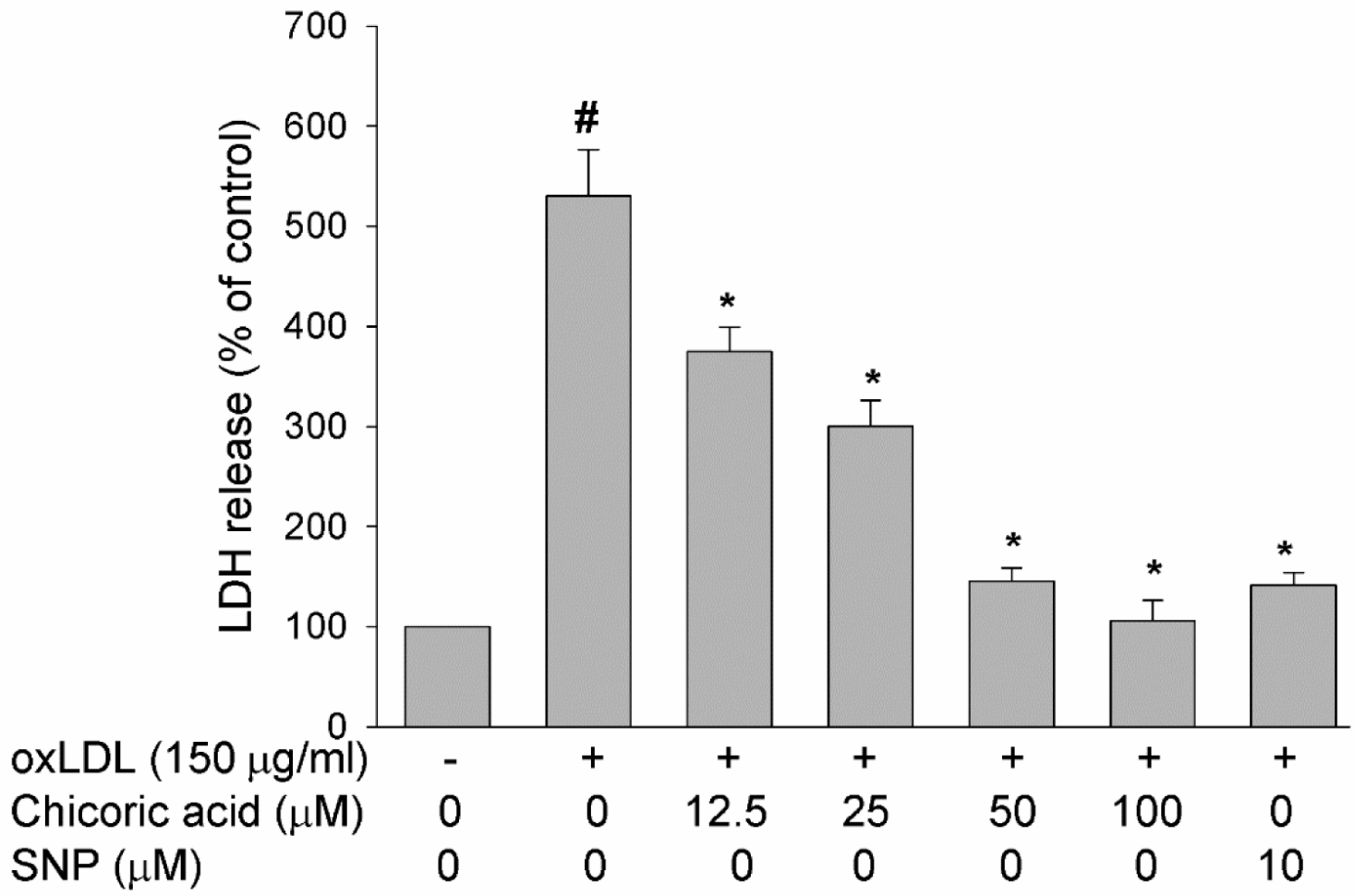

Figure 6: Effect of chicoric acid on oxLDL-reduced cell viability. HUVECs were incubated with oxLDL (150 $\mu \mathrm{g} / \mathrm{ml})$ in the absence and presence of indicated concentrations of chicoric acid or SNP (NO donor). A. Viability was determined via MTT assay B. and LDH release. The values represent means \pm SD from three separate experiments. $\# P<0.05$ vs. untreated control; $* P<0.05$ vs. oxLDL treatment. 
anion [28]. Here, we confirmed that intervention with chicoric acid mitigated the oxLDL-caused inactivation of both catalase and SOD and reduced ROS formation in HUVECs stimulated with oxLDL (Figure 2). We presume that the primary mechanism through which chicoric acid mitigates oxLDL-facilitated cell death of endothelial cells is its antioxidant function.

NO plays an important role in the regulation of leukocyte adhesion, vasodilation and platelet aggregation. Inhibition of $\mathrm{NO}$ production and bioavailability is considered to be the primary issue in the progression of atherosclerotic lesions [29]. Moreover, the altered rate of NO production with the enhanced removal of NO causes to an obvious inhibition in the bioavailability of NO, an occurrence observed in many vascular pathologies. NO inhibits the expression of cell surface adhesion molecules, such as VCAM-1, P-selectin and ICAM-1, and prevents both the expression of MCP-1 and reduced platelet adhesion. In this article, we demonstrated that pretreatment with chicoric acid obviously restores the suppression of the eNOS protein (Figure 3) via oxLDL stimulation,

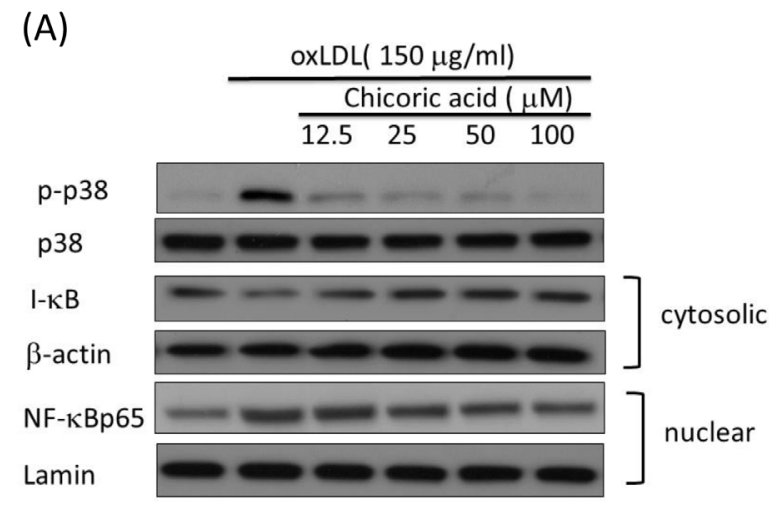

(B)

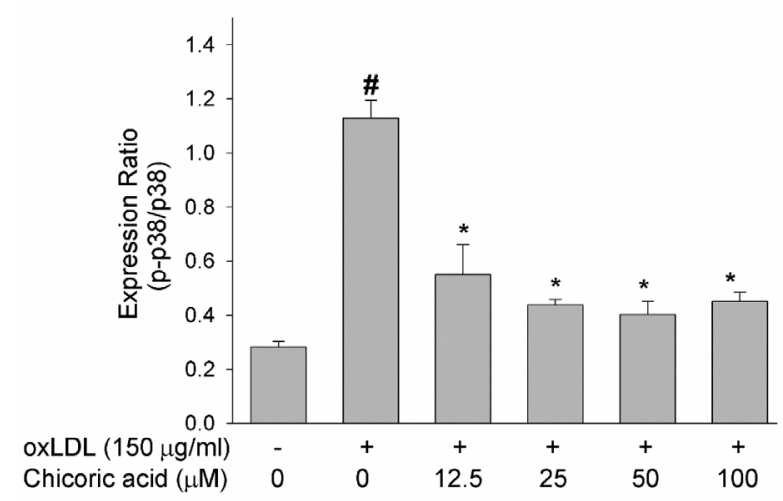

resulting in a protective effect via the prevention of oxLDL-facilitated adhesion between monocytes and HUVECs and the overexpression of adhesion molecules (Figure 7) through a mechanism potentially linked to the inhibition of oxLDL-promoted ROS formation.

There is a report that activation of $N F-\kappa B$ is accompanied by increased P53 expression [30], which subsequently facilitates a structural change in Bax that allows mitochondrial translocation of the pro-apoptotic protein Bcl-2 and facilitates the activation of caspase 3 [31]. In our study, we found that chicoric acid inhibited the translocation of NF- $\mathrm{KB}$ from the cytosol to the nucleus (Figure 7), suppressed Bax and obviously promotes the Bcl-2 expression, which prevented the activation of caspase 3 and subsequent DNA strand breaks (Figure 4).

The anti-atherosclerotic function of chicoric acid was reported previously. Martin group suggested that chicoric acid inhibits pro-inflammatory cytokine-induced overexpression of adhesion molecules and adherence of monocytes. Both of these events are highly related with atherosclerosis. They also concluded that chicoric

(C)

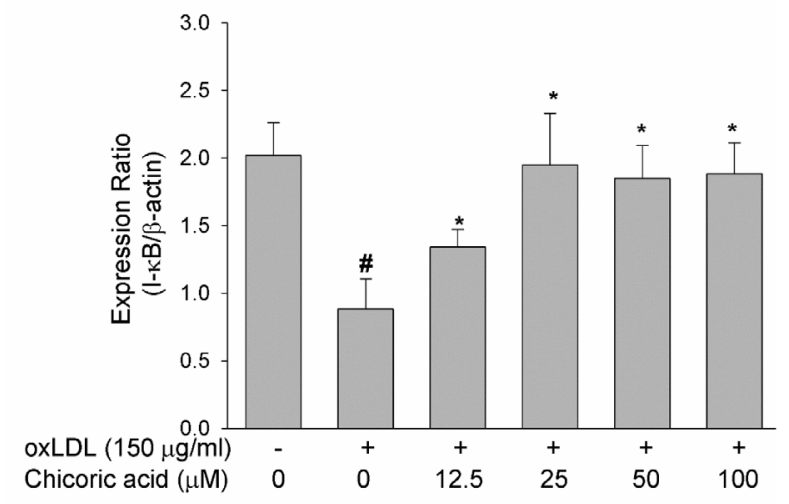

(D)

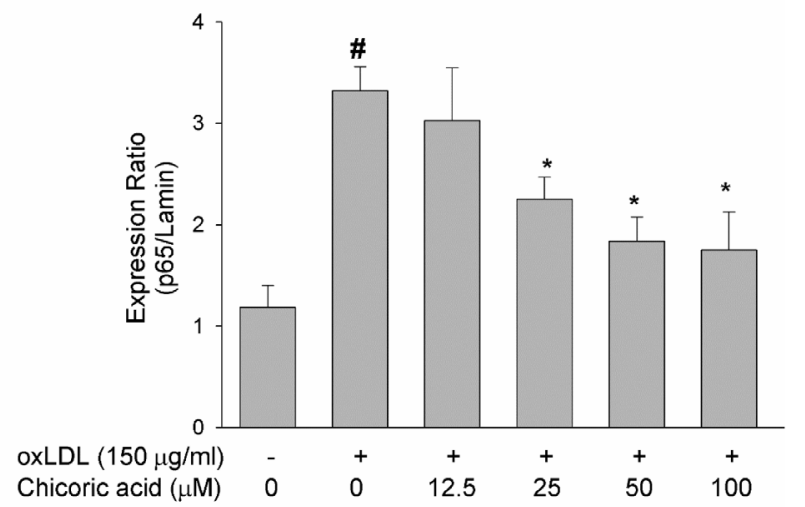

Figure 7: Effects of chicoric acid on phosphorylation of p38 MAPK and on the translocation of NF-kB. HUVECs were pretreated with indicated concentrations of chicoric acid for $2 \mathrm{~h}$ followed by exposure to oxLDL $(150 \mu \mathrm{g} / \mathrm{mL})$ for $2 \mathrm{~h} \mathrm{A.-D.} \mathrm{Western} \mathrm{blot}$ analysis was used to evaluate the expression of both phosphorylated and total p38 MAPK B., and the activation of NF-kB. Anti- $\beta$-actin and anti-Lamin antibodies were used for normalization of cytosolic and nuclear proteins $\mathbf{C}$., D., respectively. The values represent means \pm SD from three separate experiments. $\# P<0.05$ vs. untreated control; ${ }^{*} P<0.05$ vs. oxLDL treatment. 
(A)

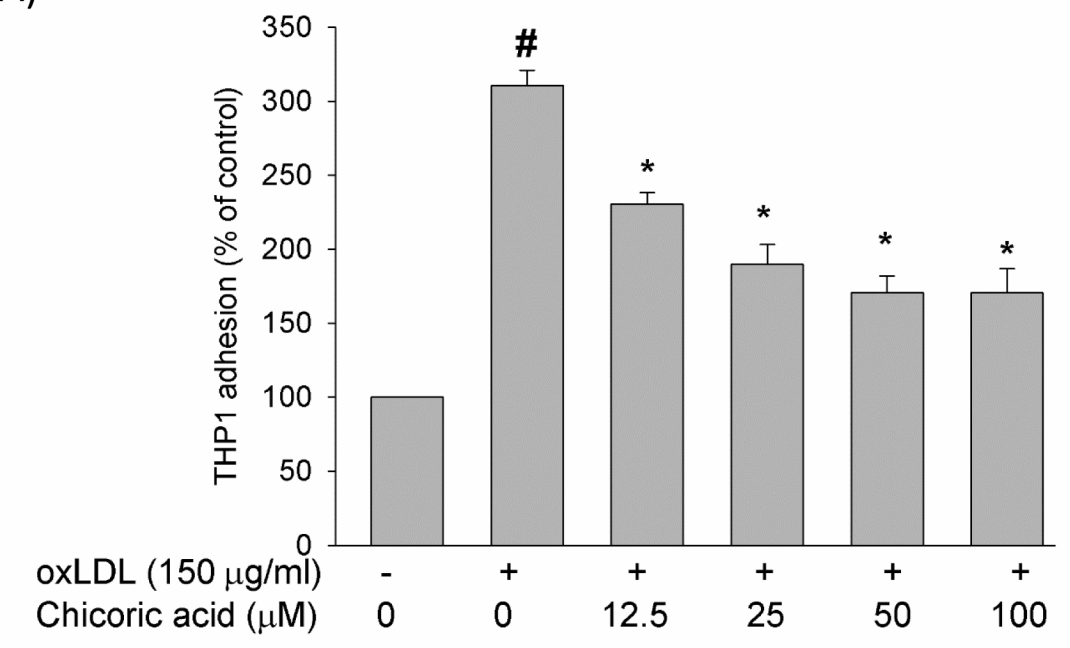

(B)

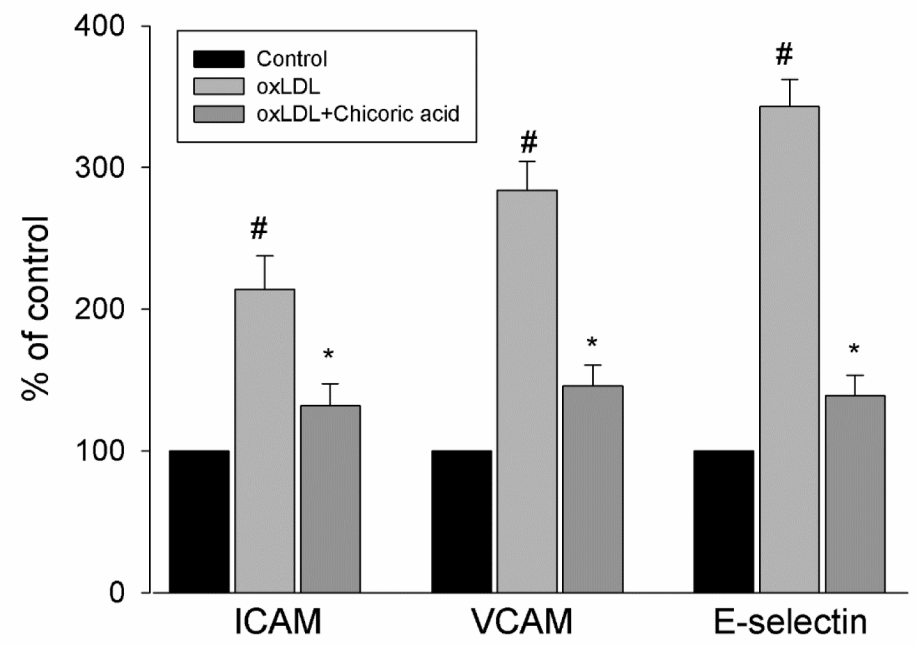

Figure 8: Effects of chicoric acid on oxLDL-induced adhesiveness of HUVECs to THP-1 monocytic cells and adhesion molecule expression. Cells were incubated with indicated concentrations of chicoric acid for $2 \mathrm{~h}$ and then incubated with ox-LDL for an additional $24 \mathrm{~h}$. A. dose-dependent effect of chicoric acid on oxLDL -induced adhesiveness of HUVECs to THP-1 was measured as described in materials and methods. HUVECs were incubated with oxLDL in the absence (control) or presence (oxLDL + chicoric acid) of $100 \mu \mathrm{M}$ chicoric acid for $24 \mathrm{~h}$. The histogram of cell surface expression of B. VCAM-1, C. ICAM-1, and D. E-selectin was generated by flow cytometry. (left) No treatment; (middle) oxLDL; (right) oxLDL + chicoric acid. The values represent means $\pm \mathrm{SD}$ from three separate experiments. $\# P<0.05$ vs. untreated control; $* P<0.05$ vs. oxLDL treatment.

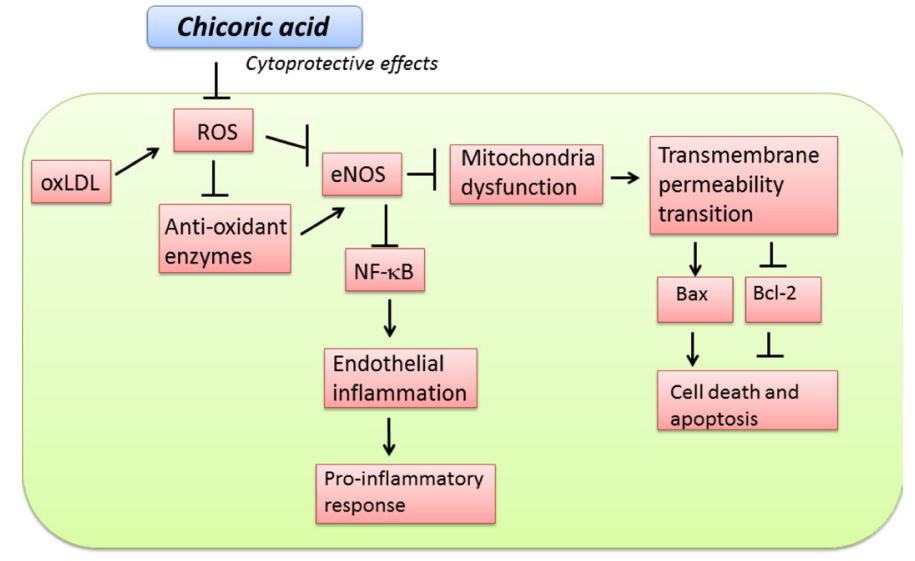

Figure 9: Schematic diagram showing cytoprotective signaling of chicoric acid in oxLDL-induced oxidative injuries in endothelial cells. The $(\rightarrow)$ indicates activation or induction, and $(-\mid)$ indicates inhibition or blockade. 
acid may be a prudent compound for prevention of cardiovascular disease [32]. The conclusion of this paper is very similar with our study, we confirmed that chicoric acid protects against oxLDL-induced adhesion molecules up-regulation and adherence of monocytes (Figure 8).

The concentrations $(12.5-100 \mu \mathrm{M})$ used are similar to those used in studies to test the inhibition of other oxidative stress-related events. For example, $100 \mu \mathrm{M}$ of chicoric acid has been reported to reverses insulin resistance as well as mitigates pro-inflammatory events in the glucosamine-treated HepG2 cells [33]. There is increasing interest in assessing the clinical efficacy of dietary supplements, natural extracts, antioxidants and polyphenol to improve health and prevent disease [34]. Therefore, understanding the molecular mechanisms through which a dietary antioxidant can prolong diseases is important to effectively couple these improvements with healthy lifestyle changes.

In conclusion, our findings suggest that chicoric acid mitigates oxLDL-induced endothelial dysfunction and inhibits oxLDL-facilitated ROS formation and the inactivation of antioxidant enzymes. chicoric acid repressed the adhesion molecules and the adherence of monocytes by attenuating NF- $\kappa \mathrm{B}$ activation. Chicoric acid treatment also inhibited oxLDL-facilitated endothelial apoptosis (Figure 9). It is likely that these beneficial effects contribute to the overall antiatherogenic function of chicoric acid. However, only in vitro investigations were used to tested the cytoprotective effects of chicoric acid from oxLDL-caused endothelial dysfunction. Animal study will be used to further evaluate the antiatherosclerotic effects of chicoric acid.

\section{MATERIALS AND METHODS}

\section{Reagents}

Fetal bovine serum, trypsin-EDTA, and M199 were bought from Gibco (Grand Island, NY, USA); low serum growth supplement (LSGS) was bought from Cascade (Portland, OR); Chicoric acid, 2', 7'-bis-2-carboxyethyl-5 (and -6)-carboxyfluorescein-acetoxymethyl ester (BCECF-AM), ethylene diaminotetraacetic acid (EDTA), 2',7'-dichlorofluorescein acetoxymethyl ester (DCFAM), 5,58,6,68-tetraethylbenzimidazolcarbocyanine iodide (JC-1), SNP, streptomycin and penicillin were bought from Sigma (St. Louis, MO); the TUNEL assay kit was bought from Boehringer Mannheim (Mannheim, Germany); the superoxide dismutase activity assay kit was bought from Calbiochem and catalase activity assay kit were bought from Abcam; active caspase 3 assay kit was obtained from BioVision (Palo Alto, CA); antiintercellular adhesion molecules (ICAM-1), anti-vascular cell adhesion molecule-1 (VCAM-1), anti-E-selectin and the annexin $\mathrm{V}$ apoptosis kit were obtained from $\mathrm{R} \& \mathrm{D}$ Systems (Minneapolis, MN); anti-eNOS, anti-p-eNOS, anti-Bcl 2 and anti-Bax were obtained from Transduction Laboratories (CA, USA).

\section{Cell culture}

Human umbilical vein endothelial cells (HUVECs), obtained from ATCC (ATCC $\AA$ PCS-100-010), were cultured in 199 medium with a low serum growth supplement. Culture dishes were coated with gelatin for 2 hours. Penicillin and streptomycin were used as an antibiotic. Trypsin-EDTA was used for cell passage. THP1 were cultured in RPMI with $10 \%$ FBS at a density of 2 to $5 \times 10^{6}$ cells $/ \mathrm{ml}$, as suggested in the product specification sheet provided by the vendor.

\section{Lipoprotein oxidation}

Human plasma LDL was purchased from Sigma. $\mathrm{CuSO}_{4}(10 \mu \mathrm{M})$ were used to oxidase LDL. After oxidation, $\mathrm{CuSO}_{4}$ was removed by PD10 columns (GE). The oxidative status of LDL were confirmed by thiobarbituric acid reactive substances (TBARS) assay.

\section{Immunoblotting}

Endothelial cells were pretreated with chicoric acid for 2 hours, and then treated with oxLDL for 24 hours. At the end of treatment, the cytosolic/nuclear protein fractions of endothelial cells were isolated by a Cytoplasmic Extraction kit, dependent on the manufacturer's instructions (PIERCE, Rockford, IL). Total protein determined by SDS-PAGE and an immunoblot assay. The blots were incubated with $5 \%$ milk for 1 hour and then incubated with primary antibodies 1 hour at room temperature respectively, followed by incubation with horseradish peroxidase-conjugated secondary antibody for 1 hour. An enhanced chemiluminescent assay (ECL; Amersham, Berkshire, UK) was used to detect the bound immunoproteins. The intensities of protein expressions were quantified by densitometric analysis (Digital Protein DNA Imagineware, Huntington Station, NY). Protein expressions were normalized by internal control genes and indicated by bar-chart.

\section{Investigation of cytotoxicity and indices of apoptosis}

To test the effect of chicoric acid on oxLDLfacilitated cytotoxicity, endothelial cells were treated with different concentrations of chicoric acid for 2 hours and then stimulated with $150 \mu \mathrm{g} / \mathrm{ml}$ oxLDL for a further 24 hours. MTT assay was used to test cell viability. Annexin 
$\mathrm{V}$ assay and the terminal deoxynucleotidyl transferasemediated dUTP nick end-labeling (TUNEL) assay were used to determine apoptotic cells by flow cytometry.

\section{ROS formation assay}

Endothelial cells were incubated in 96-well. Confluent endothelial cells were pre-treated with chicoric acid for 2 hours. oxLDL was then incubated to the medium with or without chicoric acid. The fluorescence microplate reader was used to analyze fluorescence intensity by an excitation at $540 \mathrm{~nm}$ and an emission at $590 \mathrm{~nm}$. This formula $\left[\left(\mathrm{Ft}_{2}-\mathrm{Ft}_{0}\right) / \mathrm{Ft}_{0}\right] \mathrm{X} 100$, was used to calculate the percentage increase of fluorescence $\left(\mathrm{Ft}_{2}\right.$ is the fluorescence at 2 hours of oxLDL exposure and $\mathrm{Ft} 0$ is the fluorescence at 0 hour of oxLDL exposure).

\section{Measurement of antioxidant enzyme activity}

Confluent HUVECs were pre-treated with chicoric acid for 2 hours. oxLDL was then incubated to the medium with or without chicoric acid. SOD and catalase activity were tested via an enzymatic assay method using a commercial kit dependent on the manufacturer's instructions.

\section{Expression level of adhesion molecules}

Confluent HUVECs were pre-treated with chicoric acid for 2 hours. oxLDL was then incubated to the medium with or without chicoric acid. After stimulation, endothelial cells were collected and incubated with FITCconjugated anti-body for $30 \mathrm{~min}$ at room temperature. The intensities of fluorescence intensity was quantified by flow cytometry.

\section{Investigation of mitochondrial transmembrane potential}

The mitochondrial transmembrane potential $(\Delta \Psi \mathrm{m})$ were tested by JC-1. After stimulation of oxLDL for 24 hours, endothelial cells were collected and JC-1 $(5 \mu \mathrm{M})$ was loaded for $30 \mathrm{~min}$. Investigation of the $\Delta \Psi \mathrm{m}$ was carried out by flow cytometer.

\section{Investigation of active caspase-3}

To test the effects of chicoric acid on oxLDL-caused caspase-3 activation, HUVECs were pre-treated with chicoric acid for 2 hours. oxLDL was then incubated to the medium with or without chicoric acid. The status of active caspase- 3 was investigated by flow cytometry using a commercial CaspGLOWTM Red Active Caspase-3 Staining Kit (BioVision).

\section{Monocytes adherence assay}

HUVECs were pre-treated with chicoric acid for 2 hours. oxLDL was then incubated to the medium with or without chicoric acid. THP-1 cells were labeled by BCECF-AM $4 \mu \mathrm{M}$. At the end of stimulation, medium were removed and $0.1 \mathrm{~mL} /$ well of THP-1 cells were incubated for $1 \mathrm{~h}$. The cells were allowed to adhere at 37 ${ }^{\circ} \mathrm{C}$ for 1 hour in a $5 \% \mathrm{CO}_{2}$ incubator. Non-adherent cells were washed by PBS. The adherent cells were lysed by $100 \mu 1$ PBS with $0.25 \%$ Triton X-100. The fluorescence microplate reader was used to measure the fluorescence intensity by an $485 \mathrm{~nm}$ (excitation) and $538 \mathrm{~nm}$ (emission).

\section{Analyses}

Results are expressed as mean \pm SD. Differences between the groups were analyzed using one-way ANOVA followed by post-hoc tests. A $P$-value $<0.05$ was considered statistically significant.

\section{ACKNOWLEDGMENTS}

This study was supported by grants from Ministry of Science and Technology (MOST 105-2311-B-006-008, MOST 105-2320-B-039-003-MY2 and 105-2815-C-039020-B). CMU105-S-45 and CMU105-SR-19 from China Medical University.

\section{CONFLICTS OF INTEREST}

None.

\section{REFERENCES}

1. Lum H, Roebuck KA. Oxidant stress and endothelial cell dysfunction. American journal of physiology Cell physiology. 2001; 280:C719-741.

2. Tsai KL, Chen LH, Chiou SH, Chiou GY, Chen YC, Chou HY, Chen LK, Chen HY, Chiu TH, Tsai CS, Ou HC, Kao CL. Coenzyme Q10 suppresses oxLDL-induced endothelial oxidative injuries by the modulation of LOX-1-mediated ROS generation via the AMPK/PKC/NADPH oxidase signaling pathway. Mol Nutr Food Res. 2011; 55 Suppl 2:S227-240.

3. Ou HC, Hsieh YL, Yang NC, Tsai KL, Chen KL, Tsai CS, Chen IJ, Wu BT, Lee SD. Ginkgo biloba extract attenuates oxLDL-induced endothelial dysfunction via an AMPKdependent mechanism. J Appl Physiol (1985). 2013; 114:274-285.

4. Ross R. The pathogenesis of atherosclerosis: a perspective for the 1990s. Nature. 1993; 362:801-809.

5. Ou HC, Lee WJ, Lee IT, Chiu TH, Tsai KL, Lin CY, Sheu WH. Ginkgo biloba extract attenuates oxLDL-induced 
oxidative functional damages in endothelial cells. J Appl Physiol (1985). 2009; 106:1674-1685.

6. Tsai KL, Huang YH, Kao CL, Yang DM, Lee HC, Chou HY, Chen YC, Chiou GY, Chen LH, Yang YP, Chiu TH, Tsai CS, Ou HC, et al. A novel mechanism of coenzyme Q10 protects against human endothelial cells from oxidative stress-induced injury by modulating NO-related pathways. The Journal of nutritional biochemistry. 2012; 23:458-468.

7. Cominacini L, Pasini AF, Garbin U, Davoli A, Tosetti ML, Campagnola M, Rigoni A, Pastorino AM, Lo Cascio V, Sawamura T. Oxidized low density lipoprotein (oxLDL) binding to ox-LDL receptor-1 in endothelial cells induces the activation of NF-kappaB through an increased production of intracellular reactive oxygen species. The Journal of biological chemistry. 2000; 275:12633-12638.

8. Dimmeler S, Zeiher AM. Reactive oxygen species and vascular cell apoptosis in response to angiotensin II and pro-atherosclerotic factors. Regulatory peptides. 2000; 90:19-25.

9. Robbesyn F, Salvayre R, Negre-Salvayre A. Dual role of oxidized LDL on the NF-kappaB signaling pathway. Free Radic Res. 2004; 38:541-551.

10. Hajra L, Evans AI, Chen M, Hyduk SJ, Collins T, Cybulsky MI. The NF-kappa B signal transduction pathway in aortic endothelial cells is primed for activation in regions predisposed to atherosclerotic lesion formation. Proc Natl Acad Sci U S A. 2000; 97:9052-9057.

11. Brand K, Page S, Rogler G, Bartsch A, Brandl R, Knuechel R, Page M, Kaltschmidt C, Baeuerle PA, Neumeier D. Activated transcription factor nuclear factor-kappa B is present in the atherosclerotic lesion. J Clin Invest. 1996; 97:1715-1722.

12. Salvayre R, Auge N, Benoist H, Negre-Salvayre A. Oxidized low-density lipoprotein-induced apoptosis. Biochim Biophys Acta. 2002; 1585:213-221.

13. Walpola PL, Gotlieb AI, Cybulsky MI, Langille BL. Expression of ICAM-1 and VCAM-1 and monocyte adherence in arteries exposed to altered shear stress. Arterioscler Thromb Vasc Biol. 1995; 15:2-10.

14. Azay-Milhau J, Ferrare K, Leroy J, Aubaterre J, Tournier M, Lajoix AD, Tousch D. Antihyperglycemic effect of a natural chicoric acid extract of chicory (Cichorium intybus L.): a comparative in vitro study with the effects of caffeic and ferulic acids. J Ethnopharmacol. 2013; 150:755-760.

15. Xiao H, Wang J, Yuan L, Xiao C, Wang Y, Liu X. Chicoric acid induces apoptosis in 3T3-L1 preadipocytes through ROS-mediated PI3K/Akt and MAPK signaling pathways. J Agric Food Chem. 2013; 61:1509-1520.

16. Landmann M, Kanuri G, Spruss A, Stahl C, Bergheim I. Oral intake of chicoric acid reduces acute alcohol-induced hepatic steatosis in mice. Nutrition. 2014; 30:882-889.

17. Liu Q, Chen Y, Shen C, Xiao Y, Wang Y, Liu Z, Liu $X$. Chicoric acid supplementation prevents systemic inflammation-induced memory impairment and amyloidogenesis via inhibition of NF-kappaB. FASEB J. 2016.

18. Schmitt A, Salvayre R, Delchambre J, Negre-Salvayre A. Prevention by alpha-tocopherol and rutin of glutathione and ATP depletion induced by oxidized LDL in cultured endothelial cells. British journal of pharmacology. 1995; 116:1985-1990.

19. Vassort G, Turan B. Protective role of antioxidants in diabetes-induced cardiac dysfunction. Cardiovascular toxicology. 2010; 10:73-86.

20. Mukherjee S, Coaxum SD, Maleque M, Das SK. Effects of oxidized low density lipoprotein on nitric oxide synthetase and protein kinase $\mathrm{C}$ activities in bovine endothelial cells. Cell Mol Biol (Noisy-le-grand). 2001; 47:1051-1058.

21. Vindis C, Elbaz M, Escargueil-Blanc I, Auge N, Heniquez A, Thiers JC, Negre-Salvayre A, Salvayre R. Two distinct calcium-dependent mitochondrial pathways are involved in oxidized LDL-induced apoptosis. Arteriosclerosis, thrombosis, and vascular biology. 2005; 25:639-645.

22. Cheng J, Cui R, Chen CH, Du J. Oxidized low-density lipoprotein stimulates p53-dependent activation of proapoptotic Bax leading to apoptosis of differentiated endothelial progenitor cells. Endocrinology. 2007; 148:2085-2094.

23. Chen XP, Zhang TT, Du GH. Lectin-like oxidized lowdensity lipoprotein receptor-1, a new promising target for the therapy of atherosclerosis? Cardiovasc Drug Rev. 2007; 25:146-161.

24. Ghosh S, Baltimore D. Activation in vitro of NF-kappa B by phosphorylation of its inhibitor I kappa B. Nature. 1990; 344:678-682.

25. Urban MB, Schreck R, Baeuerle PA. NF-kappa B contacts DNA by a heterodimer of the p50 and p65 subunit. Embo J. 1991; 10:1817-1825.

26. Kim JA, Territo MC, Wayner E, Carlos TM, Parhami F, Smith CW, Haberland ME, Fogelman AM, Berliner JA. Partial characterization of leukocyte binding molecules on endothelial cells induced by minimally oxidized LDL. Arteriosclerosis and thrombosis. 1994; 14:427-433.

27. Kuo WW, Huang CY, Chung JG, Yang SF, Tsai KL, Chiu TH, Lee SD, Ou HC. Crude extracts of Solanum lyratum protect endothelial cells against oxidized low-density lipoprotein-induced injury by direct antioxidant action. Journal of vascular surgery. 2009; 50:849-860.

28. Jewett SL, Rocklin AM, Ghanevati M, Abel JM, Marach JA. A new look at a time-worn system: oxidation of CuZnSOD by H2O2. Free radical biology \& medicine. 1999; 26:905-918.

29. Kurowska EM. Nitric oxide therapies in vascular diseases. Current pharmaceutical design. 2002; 8:155-166.

30. Dentelli P, Rosso A, Zeoli A, Gambino R, Pegoraro L, Pagano G, Falcioni R, Brizzi MF. Oxidative stress-mediated mesangial cell proliferation requires $\mathrm{RAC}-1 /$ reactive oxygen species production and beta4 integrin expression. 
The Journal of biological chemistry. 2007; 282:2610126110 .

31. Hoffmann J, Haendeler J, Aicher A, Rossig L, Vasa M, Zeiher AM, Dimmeler S. Aging enhances the sensitivity of endothelial cells toward apoptotic stimuli: important role of nitric oxide. Circulation research. 2001; 89:709-715.

32. Martin KR. The bioactive agent ergothioneine, a key component of dietary mushrooms, inhibits monocyte binding to endothelial cells characteristic of early cardiovascular disease. Journal of medicinal food. 2010; 13:1340-1346.
33. Zhu D, Wang Y, Du Q, Liu Z, Liu X. Cichoric Acid Reverses Insulin Resistance and Suppresses Inflammatory Responses in the Glucosamine-Induced HepG2 Cells. J Agric Food Chem. 2015; 63:10903-10913.

34. Butt MS, Imran A, Sharif MK, Ahmad RS, Xiao H, Imran M, Rsool HA. Black tea polyphenols: a mechanistic treatise. Critical reviews in food science and nutrition. 2014; 54:1002-1011. 